\section{Validación y generación de ecuaciones para estimar masa grasa corporal en adultos chilenos, formuladas a partir de bioimpedanciometría, en un amplio rango de edad e índice de masa corporal}

\author{
INGRID SCHIFFERLI I,2,a,b, JUAN JOSÉ ORELLANA-CÁCERES ${ }^{1,3, \mathrm{~b}}$, \\ GLADYS MORALES ${ }^{1,2, b}$, JORGE INOSTROZA ${ }^{4, c}$, \\ FERNANDO CARRASCO ${ }^{4}$
}

\section{Equations to estimate body composition using bioelectrical impedance in Chilean adults}

Background: Equations for the evaluation of fat-free mass (FFM) and fat mass (FM) with Bioelectrical impedance analysis (BIA) were formulated in Caucasian populations. International recommendations suggest that population-specific equations should be formulated. Aim: To validate an equation previously formulated in Chileans adults and compare it to a new equation generated on an independent sample. Material and Methods: In108 adult volunteers aged $38.1 \pm 14.1$ years ( $44 \%$ males), with a body mass index (BMI) of $25.1 \pm 4.1 \mathrm{~kg} / \mathrm{m}^{2}$, body composition was measured by BIA (Bodystat) and dual $X$-ray absorptiometry (DXA: Lunar Prodigy). Body composition estimated using Schifferli equation and BIA were compared with DEXA, by the Bland-Altman method and simple linear regression. Results: FFM and FM measured by DXA were $45.2 \pm 9.8 \mathrm{~kg}$ and $29.6 \pm 11.7 \%$ respectively. Resistance was $467.7 \pm 76.3$ ohm. Schifferli equation and BIA significantly overestimated FFM by 7.3 and $7.4 \mathrm{~kg}$, respectively. The error was higher for high levels of FFM (slope $\beta<1$, $p<0.01$ ). Both equations underestimated FM measured by DXA (averages of 7.5 and $7.8 \%$, respectively, $p<0.01$ ), without a differential bias for Schifferli equation, but with a bias in low levels of FM measured with BIA (slope $\beta<1$, $p<0.01$ ). Estimation biases could be eliminated using the regression coefficients. Conclusions: Both equations behave similarly and have biases, although less with Schifferli. Statistically correcting for biases, the new adjusted equations provide clinically valid estimates of FFM and FM. Equations should not only be population-specific, but also device-specific.

(Rev Med Chile 2020; 148: 1435-1444)

Key words: Dual-Energy X-Ray Absorptiometry; Adipose Tissue; Body Composition; Electric Impedance.

4 l estado nutricional es parte fundamental del estado de salud de un individuo. Un exceso de masa grasa corporal, se relaciona con mayor riesgo de presentar enfermedades crónicas no transmisibles, como diabetes mellitus tipo 2,
'Departamento de Salud Pública, Facultad de Medicina, Universidad de La Frontera, Temuco, Chile.

${ }^{2}$ Centro de Investigación en Epidemiología Cardiovascular y Nutricional (EPICYN), Universidad de la Frontera, Temuco, Chile. ${ }^{3}$ Centro de Capacitación, Investigación y Gestión para la Salud Basada en Evidencia (CIGES), Temuco, Chile. ${ }^{4}$ Departamento de Nutrición, Facultad de Medicina, Universidad de Chile, Santiago, Chile.

${ }^{\mathrm{a} M s c}, \mathrm{PhD}$ (c).

${ }^{\mathrm{b}} \mathrm{Msc}, \mathrm{PhD}$.

‘Tecnólogo Médico.

Fuente de financiamiento: Proyecto DIUFRO DI - 160092, Universidad de la Frontera. La organización no tuvo influencia en el diseño del estudio, recolección, análisis o interpretación de los datos; en la preparación, revisión o aprobación del manuscrito. Los autores declaran no tener conflictos de interés

Recibido el 16 de abril de 2020, aceptado el 19 de octubre de 2020.

Correspondencia a: Ingrid Schifferli Castro, ingrid.schifferli@ufrontera.cl enfermedades cardiovasculares y algunos tipos de cáncer (mama y colo-rectal, entre otros) ${ }^{1,2}$. Por ello es importante la evaluación de la composición corporal, siendo indispensable determinar de manera confiable su porcentaje de masa grasa (MG). 
Existen diferentes métodos para evaluar la composición corporal en adultos, los que pueden definirse como métodos directos, indirectos y doblemente indirectos. Éstos se basan en modelos que dividen al organismo en 2, 3 o 4 compartimentos $^{3-5}$. La bioimpedanciometría (BIA) es un método doblemente indirecto, que se basa en un modelo de dos compartimentos identificando MG y masa libre de grasa (MLG). Se define como la medición de la resistencia del cuerpo a la conducción de una corriente eléctrica alterna de baja intensidad y es inversamente proporcional al contenido de agua corporal ${ }^{6}$. La estimación de composición corporal por impedancia se hace a través de ecuaciones de regresión basadas en la correlación entre la resistencia observada y mediciones del contenido de agua corporal total, densidad corporal o MLG. Estas ecuaciones son elaboradas contra métodos de referencia o gold standard, entre los que destacan la densitometría por inmersión, pletismografía por desplazamiento de aire y absorciometría dual de rayos X (DEXA). DEXA es un método indirecto, que divide al cuerpo en tres compartimentos: MG, MLG no ósea y MLG ósea. Esta técnica utiliza dos haces de rayos $\mathrm{X}$ de diferente energía, los que, al atravesar los tejidos del organismo, son atenuados de manera diferente por el tejido mineral, masa magra no ósea y $\mathrm{MG}^{7,8}$. Proporciona múltiples ventajas, destacando su alta exactitud, con un error de estimación de $1 \%$ para masa ósea y entre 1 y $3 \%$ para MG, llegando a ser considerado uno de los métodos de referencia para el análisis de MLG y $\mathrm{MG}^{9}$; sin embargo, por su elevado costo y menor accesibilidad lo hace un método impráctico en la clínica. BIA ha tenido un desarrollo cada vez mayor en los últimos años, debido a que es un método rápido, seguro, no invasivo, indoloro, de fácil ejecución y reproducible en el tiempo. Dichas características le otorgan especial utilidad en clínica y estudios poblacionales de composición corporal. Ha sido validado en sujetos sanos, demostrando buena concordancia respecto de DEXA $^{10}$. Sin embargo, una de las recomendaciones internacionales es utilizar ecuaciones población específicas, ya que se ha visto cierta inconsistencia cuando se aplican en población diferente de la cual fue desarrollada ${ }^{11}$. Estas ecuaciones deben ser validadas contra un gold standard, evidenciando la presencia de sesgo proporcional, es decir cuando se agrega o quita una cierta magnitud respecto de la medición real, así también la presencia de sesgo diferencial, cuando la magnitud del sesgo difiere a lo largo de la escala de medición real. Los objetivos de este estudio fueron: 1. Validar, respecto de DEXA, una ecuación de estimación de grasa corporal formulada previamente en adultos chilenos por Schifferli y cols ${ }^{12}$, a partir de bioimpedanciometría de monofrecuencia en un amplio rango de edad e índice de masa corporal (IMC); 2. Desarrollar nuevas ecuaciones aplicables a BIA de multifrecuencia y 3. Comparar el desempeño de las diferentes ecuaciones a partir de BIA respecto de DEXA.

\section{Material y métodos}

Se realizó un estudio analítico transversal sobre una muestra por conveniencia de voluntarios adultos chilenos de ambos sexos y un amplio rango de edad e IMC. Para identificar participantes de una etnia diferente a la chilena, como caucásica o de pueblos originarios, se consideró el tener apellidos y auto reconocimiento de la etnia, lo cual es un método validado cuando no existen determinantes biológicas específicas para su determinación ${ }^{13}$. Se excluyó del estudio a embarazadas, sujetos portadores de patologías que cursen con edema (insuficiencia renal, insuficiencia cardiaca congestiva, insuficiencia hepática, insuficiencia venosa de extremidades inferiores), mujeres en los siete días de la etapa premenstrual y sujetos con indicación de medicamentos que puedan causar edema o deshidratación. Tampoco se incluyeron sujetos con marcapaso, amputados, sujetos con prótesis metálicas en extremidades o en columna vertebral. Se clasificó a los voluntarios según diagnóstico nutricional por IMC ${ }^{14}$.

El tamaño muestral calculado fue de 114 personas, considerando una confiabilidad esperada de $90 \%$, una precisión de 3 puntos porcentuales y un nivel de confianza de $95 \%{ }^{15}$.

Las mediciones se realizaron durante el año 2016 en el Departamento de Nutrición de la Facultad de Medicina de la Universidad de Chile, en Santiago de Chile. Los voluntarios firmaron el consentimiento informado, aprobado por el Comité de Ética Científico de la Universidad de la Frontera, cumpliendo los requisitos de la Declaración de Helsinki para investigación en humanos ${ }^{16}$.

Se midió el peso en una balanza electrónica SECA ${ }^{\circledR}$, precisión de $0,1 \mathrm{~kg}(0,1-200 \mathrm{~kg})$, ropa 
ligera y sin calzado, y la estatura con un estadiómetro adosado a la balanza con precisión de 0,1 $\mathrm{cm}(60-200 \mathrm{~cm})$. Se evaluó composición corporal por BIA-BQ (BODYSTAT ${ }^{\circledR}$, QUADSCAN 4000) y DEXA (Lunar Prodigy Advance General Electric Medical Systems Corporation, Madison, WI, USA).

La validación de la ecuación Schifferli (BIAEC) y el análisis del comportamiento de BIA-BQ respecto a DEXA se hizo utilizando el método de Bland-Altman ${ }^{17,18}$, reportando además el coeficiente de correlación intraclase (CCI). El estudio de sesgo proporcional y diferencial se hizo mediante regresión lineal simple ${ }^{19}$. Se generaron nuevas ecuaciones aplicables a BIA de multifrecuencia, utilizando coeficientes de corrección de acuerdo a los sesgos que presentaban BIA-EC y BIA-BQ. En todas las pruebas se consideró un nivel de significación estadístico de 0,05 . El procesamiento y análisis de datos se realizó con el paquete estadístico Stata 16.

\section{Resultados}

Se evaluaron 48 hombres y 60 mujeres. Las características de la muestra (promedio \pm desviación estándar) son: edad 38,1 $\pm 14,1$ años, peso $67,6 \pm 11,4 \mathrm{~kg}$, IMC $25,1 \pm 4,1 \mathrm{~kg} / \mathrm{m}^{2}$, MLG DEXA $45,2 \pm 9,8 \mathrm{~kg}$, MG DEXA 29,6 $\pm 11 \%$, resistencia (R) 467,7 \pm 76,3 ohms, índice de resistencia (IR)
$60 \pm 14,6 \mathrm{~cm}^{2} / \mathrm{ohms}$. Solo 3 sujetos $(2,8 \%)$ tenían dos apellidos de origen caucásico, $5(4,6 \%)$ tenían un apellido caucásico y solo 1 voluntario $(0,9 \%)$ tuvo un apellido mapuche. La Tabla 1 presenta las características de la muestra del estudio previo en el cual se formuló la ecuación Schifferli en $2009^{12}$ y la muestra actual (2016), sin encontrarse diferencias estadísticamente significativas para sexo, edad, peso, IMC, MG, MLG BIA, R e IR. Hubo diferencias significativas en los promedios de $\mathrm{MG}$ y MLG DEXA ( $p<0,001)$, siendo mayor el promedio de MG y menor MLG en la muestra actual.

\section{Estimación de MLG}

En este estudio BIA-EC presenta sesgo en la estimación de MLG respecto de DEXA. En promedio sobreestimó en 7,3 kg [6,7 a 7,9; IC95\%], aumentando la sobreestimación en $0,85 \mathrm{~kg}$ por kilo de aumento de MLG [ $\beta$ : 0,81 a 0,90; IC95\%] (Figura 1). Dicho sesgo puede ser eliminado ajustando la medición por la magnitud del sesgo diferencial representado por la pendiente de la recta de regresión correspondiente, obteniéndose una nueva ecuación BIA-EC ajustada (BIA-ECa): MLG_BIA-EC ${ }^{\star} 0,8538$ (Tabla 2), cuyos resultados eliminan además el sesgo proporcional remanente al ser estadísticamente no significativo de $0,37 \mathrm{~kg}$ $[-2,0$ a 2,7; IC95\%], con CCI final de 96,4 [95,1 a 97,8; IC95\%] (Figura 2).

Al evaluar el desempeño de BIA-BQ en la

Tabla 1. Resumen estadístico según año toma de datos

\begin{tabular}{|c|c|c|c|c|c|c|c|}
\hline \multirow[t]{2}{*}{ Variables } & \multicolumn{3}{|c|}{2009} & \multicolumn{3}{|c|}{2016} & \multirow[t]{2}{*}{ Valor $p$} \\
\hline & Rango & $\mathbf{P}_{50}$ & $\bar{x} \pm s$ & Rango & $\mathbf{P}_{50}$ & $\bar{x} \pm s$ & \\
\hline Sexo (\% hombre) & & & 50,0 & & & 40,0 & $0,301 *$ \\
\hline Edad (años) & $18,0-64,0$ & 32,0 & $36,0 \pm 13,2$ & $18,0-65,0$ & 33,0 & $38,1 \pm 14,1$ & 0,311 \\
\hline Peso $(\mathrm{kg})$ & $42,0-99,0$ & 69,0 & $69,2 \pm 11,8$ & $40,5-105,3$ & 67,1 & $67,6 \pm 11,4$ & 0,325 \\
\hline $\mathrm{IMC}\left(\mathrm{kg} / \mathrm{m}^{2}\right)$ & $18,7-34,2$ & 24,6 & $25,1 \pm 3,3$ & $18,9-34,8$ & 24,2 & $25,1 \pm 4,1$ & 0,924 \\
\hline MG DEXA (\%) & $5,1-46,6$ & 22,2 & $22,9 \pm 9,1$ & $10,8-49,8$ & 30,8 & $29,6 \pm 11,7$ & 0,000 \\
\hline MLG DEXA (kg) & $33,3-83,0$ & 51,3 & $53,6 \pm 12,4$ & $29,2-69,8$ & 43,3 & $45,2 \pm 9,8$ & 0,000 \\
\hline MG BIA (\%) & $3,0-37,4$ & 21,4 & $20,4 \pm 7,4$ & $1,0-45,1$ & 21,4 & $21,8 \pm 13,3$ & 0,321 \\
\hline MLG BIA $(\mathrm{kg})$ & $31,5-81,2$ & 53,6 & $55,2 \pm 11,4$ & $32,2-83,0$ & 51,3 & $52,6 \pm 11,9$ & 0,094 \\
\hline R (ohms) & $335-693$ & 470,0 & $482,6 \pm 77,4$ & $309-660$ & 455,0 & $467,7 \pm 76,3$ & 0,143 \\
\hline IR (cm²/ohms) & $33,2-93,5$ & 55,6 & $59,1 \pm 14,0$ & $37,5-97,4$ & 57,4 & $60,0 \pm 14,6$ & 0,656 \\
\hline
\end{tabular}

P50: percentil 50; * Test exacto de Fisher. Los demás corresponden a T-test (varianzas iguales o distintas según corresponda). IMC = índice de masa corporal, $M G=$ masa grasa; $M L G=$ masa libre de grasa; $R=$ resistencia; $I R=$ índice de resistencia (estatura, $\mathrm{cm}^{2} / \mathrm{R}$ ). 


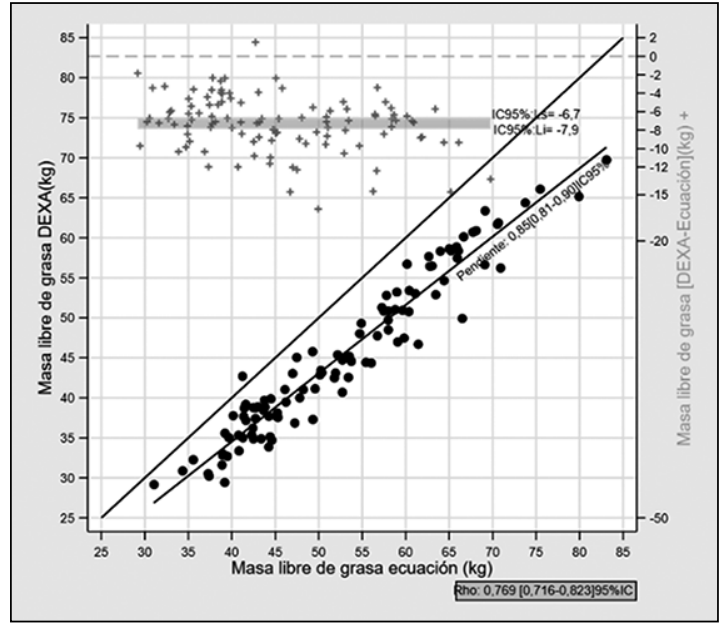

Figura 1. Masa libre de grasa DEXA versus Ecuación Schifferli (BIA-EC).

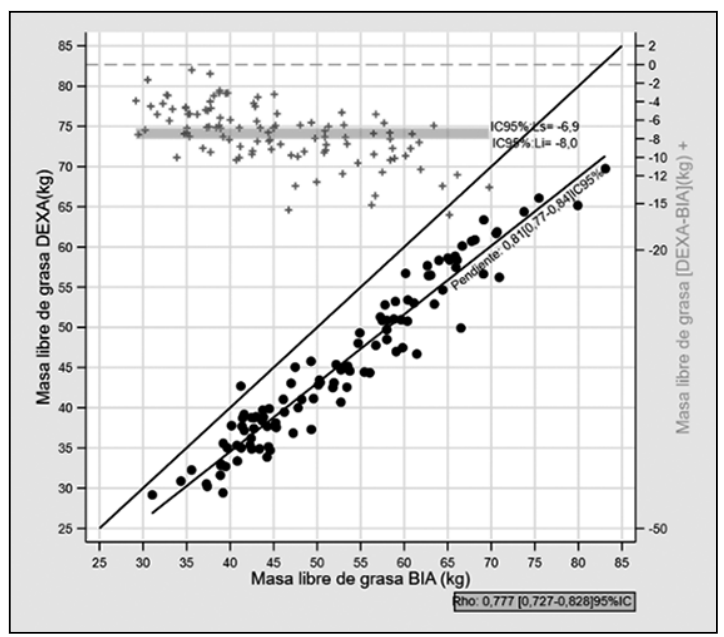

Figura 3. Masa libre de grasa DEXA versus BIA-BQ.

estimación de MLG, encontramos que también la sobreestima significativamente, en promedio $7,4 \mathrm{~kg}$ [6,9 a 8,0; IC95\%], aumentando la sobreestimación en $0,81 \mathrm{~kg}$ por kilo de aumento del nivel de MLG [ $\beta$ : 0,77 a 0,84; IC95\%] (Figura 3). $\mathrm{Al}$ ajustar la medición de BIA-BQ, primero por sesgo diferencial y luego por sesgo proporcional remanente (también estadísticamente significativo [0,97 a 4,5; IC95\%]), se obtiene la ecuación BIA-BQ ajustada: $M L G \_B I A-B Q * 0,807+2,737 \mathrm{sin}$ sesgo a la medición respecto de DEXA, con un CCI final de 97,8\% [97,0 a 98,6; IC95\%] (Figura 4).

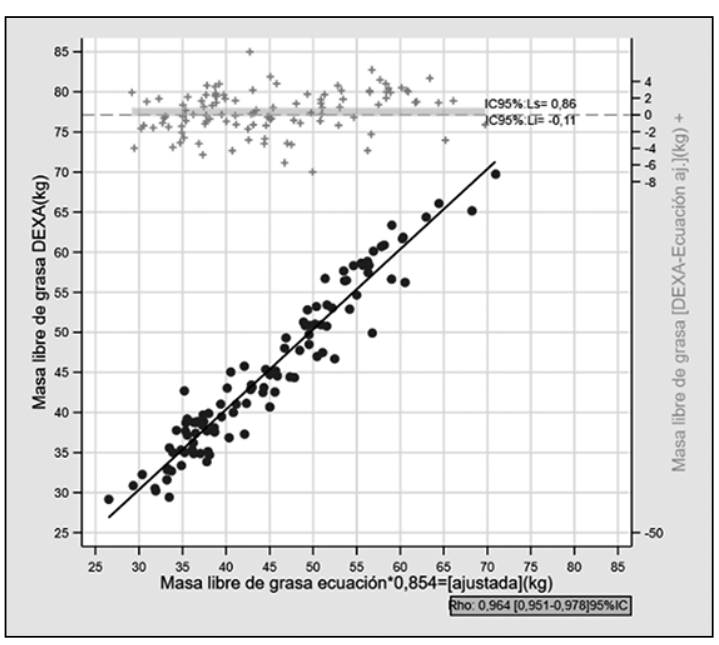

Figura 2. Masa libre de grasa DEXA versus Ecuación Schifferli (BIA-EC) ajustada por sesgo diferencial.

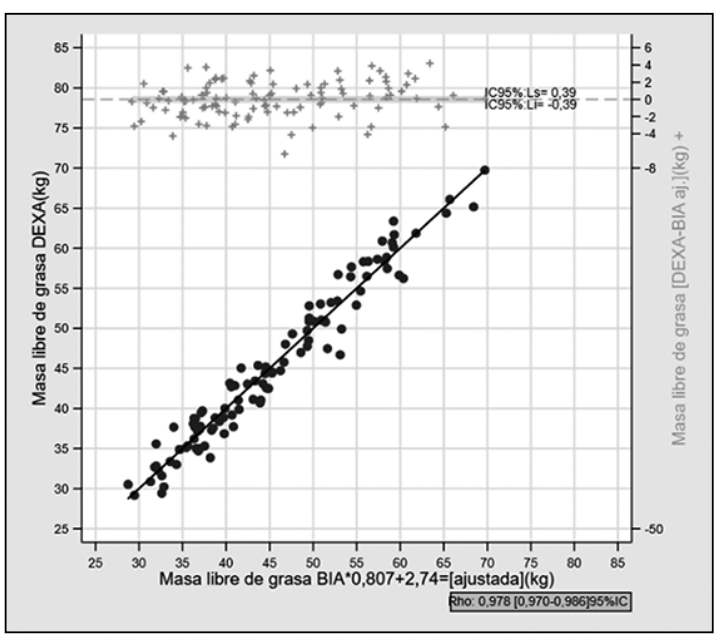

Figura 4. Masa libre de grasa $D E X A$ versus $B I A-B Q$ ajustada por sesgo diferencial y proporcional.

\section{Estimación del porcentaje de MG}

BIA-EC presenta sesgo en la estimación de MG respecto de DEXA. En promedio subestimó en 7,5 puntos porcentuales (pp) [6,7 a 8,2; IC95\%], sin sesgo diferencial a la magnitud de la medida (Figura 5). Al ajustar la medición de BIA-EC, se obtiene la ecuación BIA-EC ajustada: MG_BIAEC+7,476 (Tabla 2) sin sesgo a la medición respecto de DEXA, con un CCI final de 94,5\% [92,5 a 96,6; IC95\%] (Figura 6).

BIA-BQ también subestima la MG, significativamente, en promedio $7,8 \mathrm{pp}$ [7,1 a 8,5; IC95\%], 
aumentando la subestimación en 0,85 pp por cada pp de aumento en la MG [ $\beta$ : 0,81 a 0,90; IC95\%] (Figura 7). Al ajustar la medición de BIA-BQ, primero por el sesgo diferencial y luego por el sesgo proporcional se obtiene la ecuación BIA-BQ ajustada: $M G$ BIA-BQ ${ }^{\star} 0,853+10,991$, sin sesgo a la medición respecto de DEXA, con un CCI final de 82,2\% [78,3 a 86,1; IC95\%] (Figura 8).

La Tabla 2 presenta un ejemplo en la estimación de MLG (kg) y MG (\%) por BIA-BQ, BIA-EC,

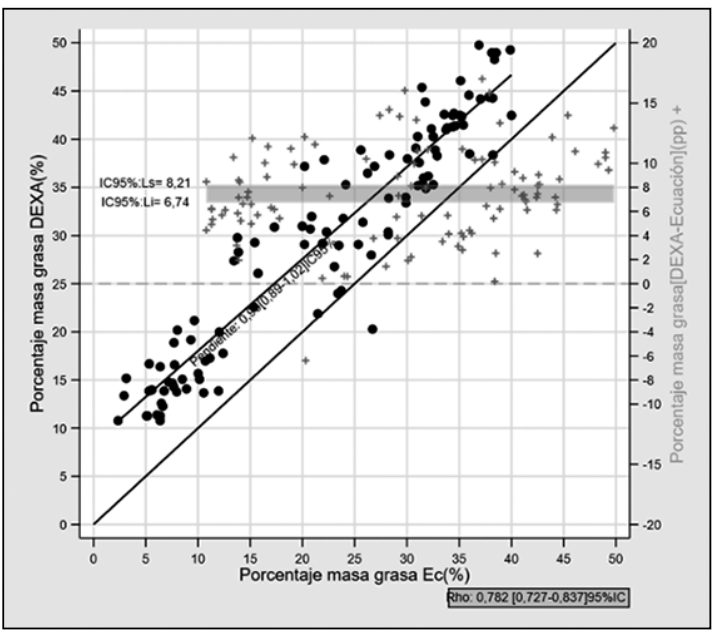

Figura 5. Porcentaje de masa grasa por DEXA versus estimado por BIA-EC. y por las ecuaciones generadas a partir de BIA de multifrecuencia ajustadas por sesgos.

La Figura 9 presenta la comparación de la validez de los métodos ajustados por sesgo para estimación de MLG según estado nutricional (EN) y sexo. En normopeso, BIA-EC sobreestima la MLG respecto de DEXA en promedio en $1,17 \mathrm{~kg}[0,59$ a 1,76; IC95\%], mientras que en BIA-BQ solo hay una diferencia promedio de $0,14 \mathrm{~kg}[-0,34$ a 0,62 ; IC95\%]. Entre ambas ecuaciones dichas diferencias

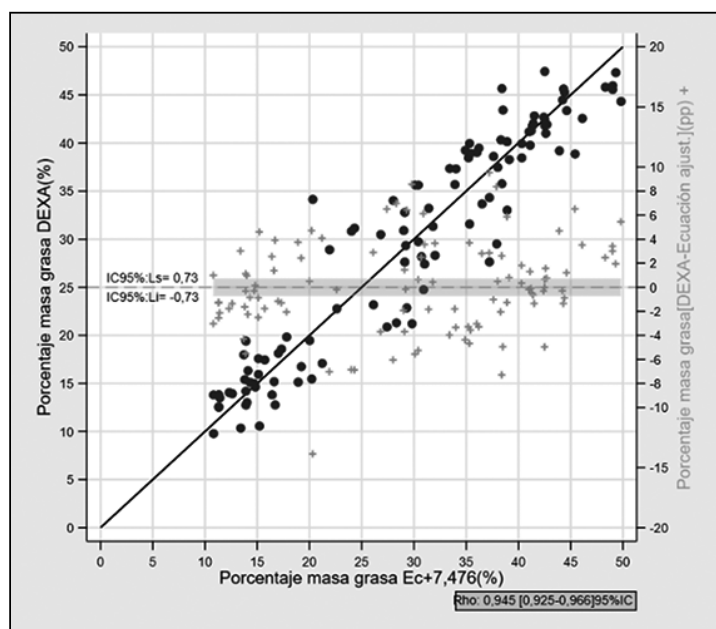

Figura 6. Porcentaje de masa grasa por DEXA versus estimado por BIA-EC ajustada por sesgo.

Tabla 2. Estimación de masa libre de grasa y masa grasa por BIA_EC y BIA_BQ ajustadas por sesgos

\begin{tabular}{|c|c|c|c|c|c|c|c|c|c|c|c|c|c|c|}
\hline & \multicolumn{14}{|c|}{ Factor de ajuste por sesgo } \\
\hline \multirow{2}{*}{$\mathrm{MLG}, \mathrm{kg}$} & \multicolumn{14}{|c|}{$\mathrm{EC} \cdot 0,854$} \\
\hline & \multicolumn{14}{|c|}{$\mathrm{BQ} \cdot 0,807+2,737$} \\
\hline \multirow{2}{*}{ MG, \% } & \multicolumn{14}{|c|}{$\mathrm{EC}+7,476$} \\
\hline & \multicolumn{14}{|c|}{$\mathrm{BQ} \cdot 0,853+10,991$} \\
\hline \multirow{2}{*}{\multicolumn{15}{|c|}{$\begin{array}{l}\text { - Ecuación Schifferli para MLG } \rightarrow \text { BIA_EC= sexo } \cdot 8,867+\text { peso } \cdot 0,369+\mathrm{IR} \cdot 0,477+\mathrm{R} \cdot 0,037-22,426 \\
\text { - } \% \mathrm{MG}=(\text { peso }-\mathrm{MLG}) / \text { peso } \cdot 100 \\
\text { - Donde } \mathrm{MLG}(\mathrm{kg}) \text {, peso }(\mathrm{kg}) \text {, estatura }(\mathrm{cm}) \text { sexo }(0: \text { mujer, } 1: \text { hombre), R: resistencia }(\mathrm{ohm}), \\
\text { IR: índice de resistencia }\left(\mathrm{cm}^{2} / \mathrm{ohm}\right) \\
\text { Ejemplo }\end{array}$}} \\
\hline & & & & & & & & & & & & & & \\
\hline \multirow{2}{*}{$\begin{array}{c}\text { Sexo } \\
\text { 1: Hombre } \\
0: \text { Mujer }\end{array}$} & \multirow{2}{*}{$\begin{array}{l}\text { Peso } \\
(\mathrm{kg})\end{array}$} & \multirow{2}{*}{$\begin{array}{l}\text { Talla } \\
(\mathrm{cm})\end{array}$} & \multirow{2}{*}{$\begin{array}{c}\mathrm{R} \\
\text { (ohm) }\end{array}$} & \multirow{2}{*}{$\begin{array}{c}\begin{array}{l}\mathrm{IR}= \\
\text { talla }\end{array} \\
\mathrm{R}\end{array}$} & \multicolumn{5}{|c|}{ MLG } & \multicolumn{5}{|c|}{$\% M G$} \\
\hline & & & & & DEXA & \multirow{2}{*}{$\begin{array}{c}\text { BIA } \\
\text { BQ } \\
48,0\end{array}$} & $\begin{array}{c}\text { BIA } \\
\text { BQ Aj. }\end{array}$ & $\begin{array}{l}\text { BIA } \\
\mathrm{EC}\end{array}$ & $\begin{array}{c}\text { BIA } \\
\text { EC Aj. }\end{array}$ & DEXA & $\mathrm{BQ}$ & BQ Aj. & $E C$ & $E C A j$ \\
\hline 0 & 63,4 & 162 & 513 & 51,2 & 39,9 & & 41,5 & 44,45 & 37,9 & 33,9 & 24,3 & 31,7 & 30,0 & 37,5 \\
\hline
\end{tabular}




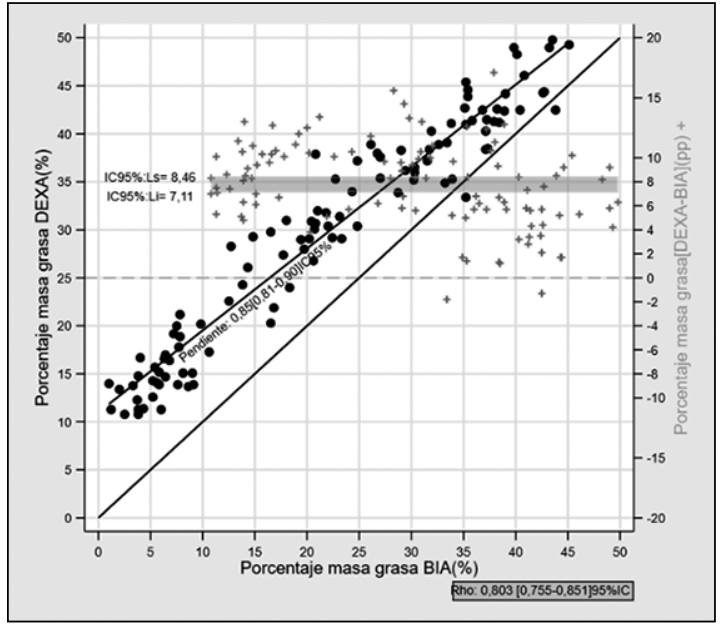

Figura 7. Porcentaje de masa grasa por DEXA versus estimado por BIA-BQ.

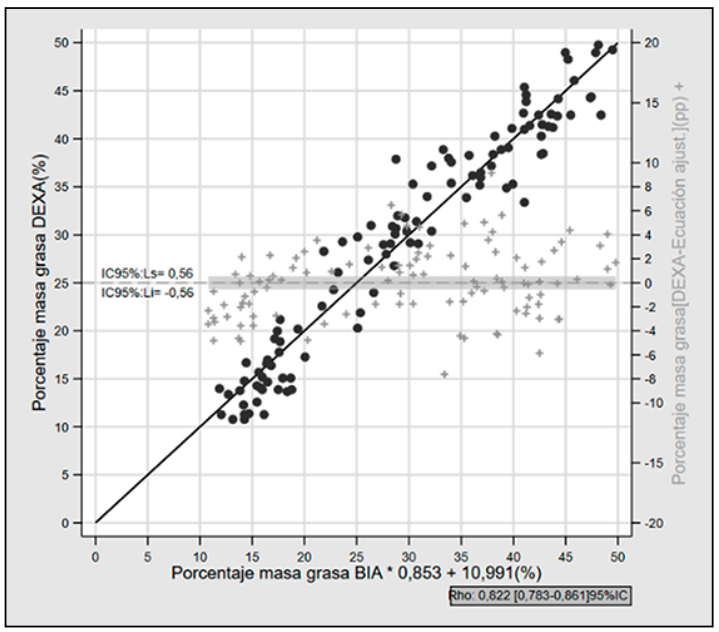

Figura 8. Porcentaje de masa grasa por DEXA versus estimado por BIA-BQ ajustada por sesgo.

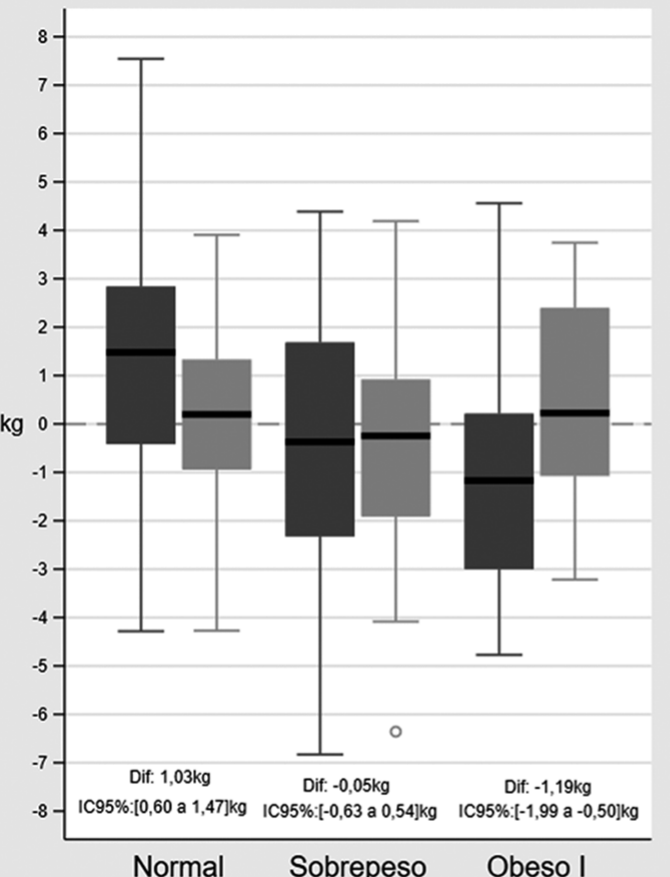

[DEXA - $\left.\left(\mathrm{Ec}^{\star} 0.854\right)\right](\mathrm{kg})$

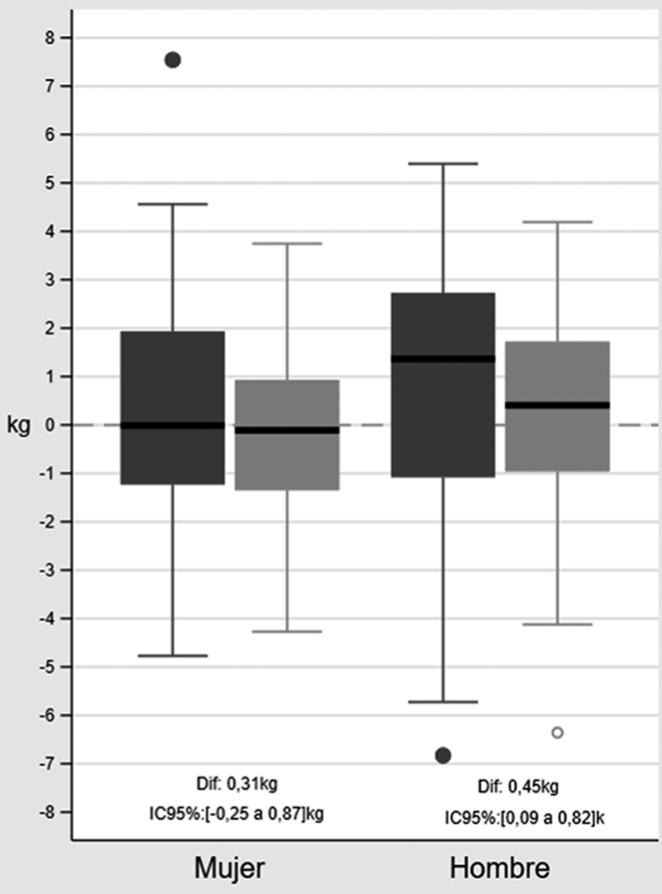

[DEXA - $\left.\left(\mathrm{BIA}^{*} 0.807+2.737\right)\right](\mathrm{kg})$

Figura 9. Diferencias absolutas de masa libre de grasa entre las estimaciones de los métodos BIA-BQ y BIA-EC ajustados por sesgo, respecto de DEXA, según sexo y diagnóstico nutricional. El intervalo de confianza (IC95\%) que no contiene el valor cero, indica hay diferencias promedio entre los métodos estadísticamente significativas. Los puntos representan valores outliers. Ec= $B I A-E C, B I A=B I A-B Q$. 
son estadísticamente significativas $[0,60$ a 1,47 ; IC95\%]. En sobrepeso, ambas ecuaciones se comportan de manera similar, subestimando la MLG en promedio en 0,49 kg BIA-EC y 0,44 kg BIA-BQ, sin diferencias significativas entre ambos métodos [-0,63 a 0,54; IC95\%]. En obesidad BIA-EC subestima la MLG en $0,88 \mathrm{~kg}$, mientras que BIA-BQ la sobreestima en $0,31 \mathrm{~kg}$, con diferencias significativas en el desempeño de la validez de ambos métodos [-1,88 a -0,50; IC95\%]. Al comparar por sexo, no se observan diferencias estadísticamente significativas en el desempeño de la validez de los métodos para estimar MLG en mujeres ( $p=0,269$, Ttest). En hombres, ambos métodos sobreestiman MLG respecto de DEXA, con mayores discrepancias para BIA-EC y con diferencias estadísticamente significativas [0,09 a 0,82; IC95\%].

La Figura 10 presenta las diferencias de MG estimadas por BIA-EC y BIA-BQ ajustadas por sesgos según EN y sexo. En normopeso, ambas ecuaciones subestiman la $\mathrm{MG}$, con valores de $-0,55$ para BIA-EC y $-0,42$ pp para BIA-BQ, sin diferencias significativas entre ambas $[-0,97$ a 0,71 ; IC95\%]. En sobrepeso, ambos métodos sobreestiman respecto de DEXA, en 0,80 para BIA-EC y 0,70 pp para BIA-BQ, sin diferencias significativas [-0,74 a 0,94; IC95\%]. En obesidad, BIA-EC sobreestima la MG en 0,47 pp y BIA-BQ en 0,21 pp respecto de DEXA, sin diferencias significativas $(\mathrm{p}=0,54, \mathrm{~T}$ test $)$.

En mujeres, BIA-EC subestima la MG en 1,04 pp y BIA-BQ en 0,26 pp, sin diferencias estadísticamente significativas ( $p=0,06, T$ test). En hombres, BIA-EC sobreestima en 1,3 pp y BIA-BQ solo en $0,32 \mathrm{pp}$, observándose diferencia significativa entre ambos valores $[0,40$ a 1,$55 ;$ IC95\%].
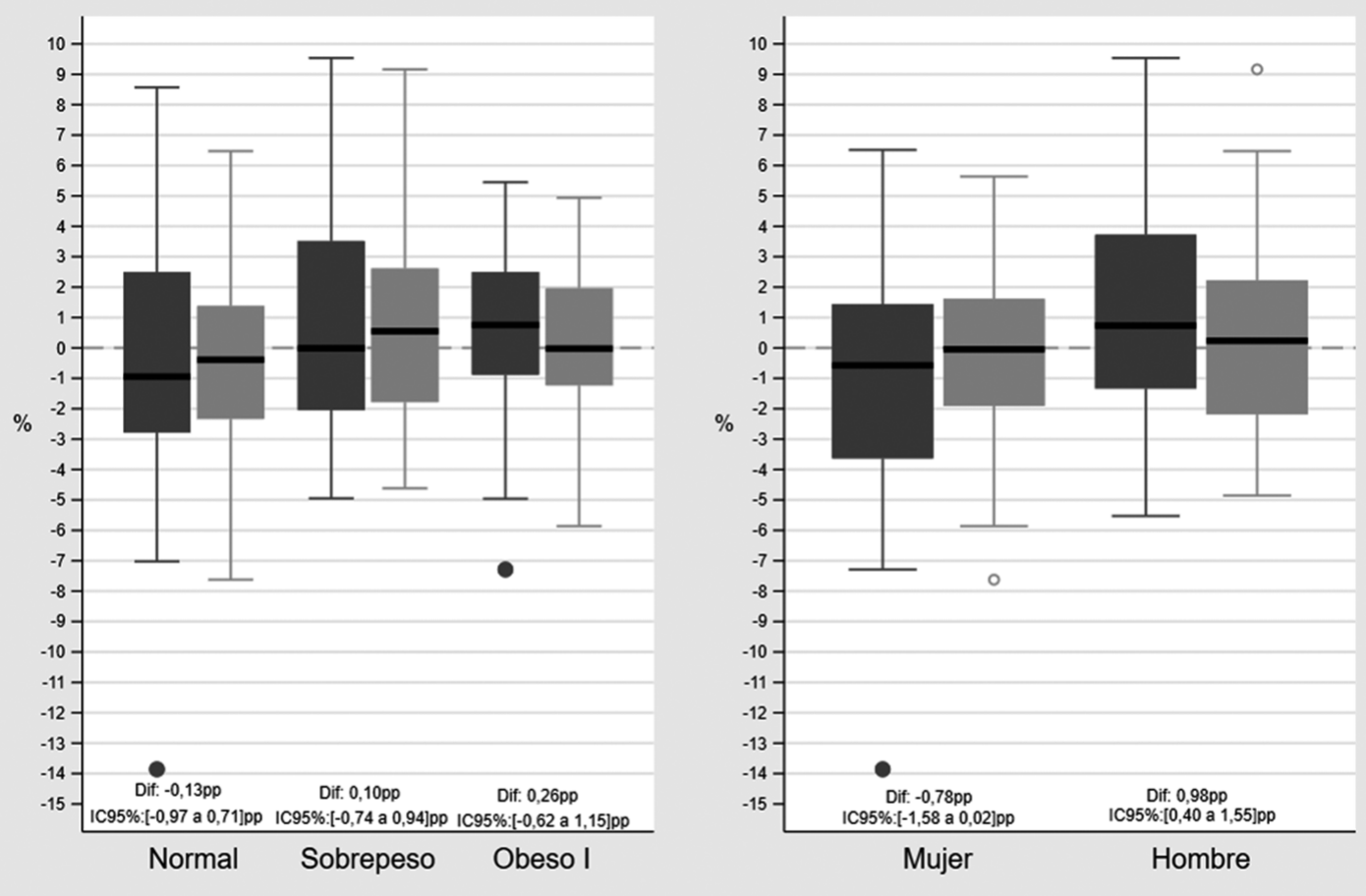

$[D E X A-(E c+8.471)](p p)$

[DEXA - $\left.\left(\mathrm{BIA}^{*} 0,853+10,991\right)\right](\mathrm{pp})$

Figura 10. Porcentaje de masa grasa por DEXA versus estimado por BIA-EC ajustada por sesgo.Diferencias absolutas de masa grasa entre las estimaciones de los métodos BIA-BQ y BIA-EC ajustados por sesgo, respecto de DEXA, según sexo y diagnóstico nutricional. El intervalo de confianza (IC95\%) que no contiene el valor cero, indica hay diferencias promedio entre los métodos estadísticamente significativas. Los puntos representan valores outliers. Ec=BIA-EC, BIA=BIA-BQ. 


\section{Discusión}

DEXA es considerado un método de referencia para la evaluación de la composición corporal a nivel clínico; sin embargo, su elevado costo y el ser un equipo no portable, impide su uso de manera rutinaria.

BIA presenta múltiples ventajas, especialmente debido a su portabilidad y fiabilidad en la medición de MLG y estimación de $\mathrm{MG}^{20}$, cuando se siguen adecuadamente protocolos en el estado de hidratación, ejercicio físico y periodo de ayuno antes de la evaluación ${ }^{21}$. Una de las limitaciones de BIA es que la mayoría de las ecuaciones predictivas han sido desarrolladas en población caucásica ${ }^{11}$ de Norteamérica y Europa. Diversos estudios han comprobado que, aplicando estas ecuaciones en individuos con características diferentes a la población de origen, el resultado ha sido inconsistente ${ }^{22,23}$.

Estudios recomiendan utilizar ecuaciones población-específicas debido principalmente a diferencias étnicas en constitución corporal y longitud de extremidades inferiores. Los errores en la estimación de MLG por diferencias étnicas pueden llegar a 3\% $\%^{7,24}$. En nuestro estudio, según el análisis basado en los apellidos, $\sim 92 \%$ de los sujetos presenta probablemente un mestizaje característico de chilenos, $\sim 7 \%$ una mayor etnicidad caucásica, y sólo $\sim 1 \%$ un mayor predominio mapuche.

Este estudio encontró que la ecuación formulada por Schifferli y cols, sobreestima la MLG en relación a DEXA, al igual que BIA-BQ, siendo mayor a medida que aumenta la unidad de medida; por ejemplo, a mayor peso, mayor sobreestimación. Debido a que ambas presentaban sesgos significativos, estos se corrigieron, generando nuevas ecuaciones ajustadas para estimación de MLG y MG aplicables a equipos BIA de multifrecuencia. $\mathrm{Al}$ analizar ambas ecuaciones respecto de DEXA, el comportamiento fue similar. En la estimación de $M G$, ambas ecuaciones subestiman el valor de DEXA, sin sesgo diferencial con BIA-EC y aumentando el sesgo en valores bajos de medición en BIA-BQ. El análisis de MLG estimada por las ecuaciones ajustadas por sesgo y considerando el estado nutricional, muestra que ambas sobreestiman la MLG respecto de DEXA en sujetos normopeso, con mejor comportamiento para BIA-BQ. En sobrepeso ambas ecuaciones subestiman MLG y en obesos BIA-EC la subestima y BIA-BQ la sobrees- tima levemente. Ambas ecuaciones subestiman la MG en normopeso, mientras que la sobreestiman en sobrepeso. En obesos solo BIA-EC subestima significativamente la MG respecto de DEXA.

El comportamiento difiere según sexo: en mujeres, para MLG, tanto BIA-EC como BIA-BQ ajustadas tienen buena concordancia con DEXA y sin diferencias significativas entre ambas; en hombres BIA-BQ se comporta mejor, aunque sin diferencias significativas. BIA-EC subestima la MG en mujeres, y en hombres, la MG es sobreestimada por ambas ecuaciones, ajustándose más en BIA-BQ.

Ambas ecuaciones presentan sesgos. Si se corrigen, arrojan estimaciones clínicamente válidas de MLG y MG. En este estudio la ecuación Schifferli se comporta similar a BIA-BQ, aunque con menos sesgos, comportamiento similar a lo encontrado en otra investigación ${ }^{25}$. Esto difiere del estudio en el cual se formuló la ecuación ${ }^{12}$, lo que lleva a plantear que las ecuaciones, además de ser población- específicas también podrían ser equipo-específicas, ya que en la muestra original (2009), se utilizó un bioimpedanciómetro de monofrecuencia (Biodynamics ${ }^{\circledR} ; 50 \mathrm{kHz}, 800 \mathrm{~mA}$ ), por tanto, menos preciso y sensible, mientras que en esta investigación se utilizó un equipo multifrecuencia (Quadscan 4000, Bodystat ${ }^{\circledR}$ ), siendo esta una limitación para el objetivo de validación de la ecuación ya publicada. Por otra parte, las características de la muestra fueron muy similares en ambos años, pero con mayor porcentaje de masa grasa en la muestra del $2016(\mathrm{p}<0,05)$.

La ecuación de Schifferli es de utilidad y aportará resultados más concordantes con el método de referencia DEXA en adultos chilenos cuando se utilicen equipos de monofrecuencia; sin embargo, no hay diferencias significativas entre esta ecuación con la incorporada en el equipo de BIA multifrecuencia Quadscan 4000 de Bodystat ${ }^{\circledR}$. Por otra parte, este estudio generó nuevas ecuaciones tanto para BIA-BQ como para BIA_EC aplicables a equipos de multifrecuencia con mayor concordancia respecto del gold standard.

En nuestro país, recientemente se está comercializando un bioimpedanciómetro de multifrecuencia validado en población caucásica, japonesa y mexicana ${ }^{26,27}$. Sería interesante validarlo en población chilena, considerando el interés clínico de conocer de manera precisa y fiable la composición corporal a nivel individual. 
Agradecimientos: A la Universidad de la Frontera, que a través del Proyecto DIUFRO DI160092, financió esta investigación.

\section{Referencias}

1. Must A, Spadano J, Goackley EH, Field AE, Gloditz G, et al. The disease burden associated with overweight and obesity. JAMA 1999; 282: 1523-29.

2. Kopelman PG. Obesity as a medical problema. Nature 2000; 404: 635-43.

3. González E. Composición corporal: estudio y utilidad clínica. Endocrinol Nutr 2013; 60 (2): 69-75.

4. Brodie D, Moscrip V, Hutcheon R. Body composition measurement: A review of hidrodensitometry, anthropometry and impedance methods. Nutrition 1998; 14: 296-310.

5. Wilson J, Strauss B, Fan B, Duewer F, Shepard J. Improved 4-compartment body-composition model for a clinically accesible measure of total body protein. Am J Clin Nutr 2013; 97: 497-504.

6. Sánchez-Iglesias A, Fernández-Lucas M, Teruel J. Fundamentos eléctricos de la bioimpedancia. Nefrología 2012; 32 (2): 133-5.

7. Daniel H. De Girolami. Métodos complementarios. En: Fundamentos de valoración nutricional y composición corporal. Argentina. Editorial El Ateneo 2003; 17: 217-51.

8. Andreoli A, Scalzo G, Masala S, Tarantino U, Guglielmi G. Body composition assessment by dual-energy X- ray absorciometry (DXA). Radiol med 2009; 114: 286-300.

9. Ellis KJ. Human body composition: in vivo methods. Phisiol Rev 2000; 80: 64-80.

10. Bellido D, Carreira J. Desarrollo de ecuaciones predictivas para el cálculo de composición corporal por impedanciometría. Rev Esp Obes 2006; 4 (2): 97-106.

11. Kyle U, Bosaeus I, De Lorenzo A, Deurenberg P, Elia M, Gómez J, et al. Bioelectrical impedance analysis-part I: review of principles and methods. Clin Nutr 2004; 23: 1226-43.

12. Schifferli I, Carrasco F, Inostroza J. Formulación de una ecuación para predecir la masa grasa corporal a partir de bioimpedanciometría en adultos en un amplio rango de edad e índice de masa corporal. Rev Med Chile 2011; 139: 1534-43.

13. Sapunar J, Bravo P, Schneider H, Jiménez M. ¿Es la etnia mapuche un factor de riesgo para padecer fractura de fémur proximal en adultos mayores? Rev Méd Chile 2003; 131: 1135-41.

14. WHO. Obesity: Preventing and managing the global epidemic. Report of WHO consultation on obesity. Geneva, 1998.
15. Streiner DL, Norman GR. Health measurement scale (p124). (Oxford University Publication, Ed.) (2nd edition). Oxford University Press. 1996.

16. World Medical Association. World Medical Association Declaration of Helsinki: Ethical Principles for Medical Research Involving Human Subjects. JAMA. 2013; 310: (20): 2191-94. Doi: 10.1001/jama.2013.281053.

17. Bland JM, Altman DG. Comparing methods of measurement: why plotting difference against standard method is misleading. Lancet 1995; 346 (8982): 1085-87.

18. Bland-Altman plot. Manual: Method comparision \& evaluation. MEDCALC ${ }^{\circledR}$. [Consultado 05 de abril de 2019]. Disponible en: https://www.medcalc.org/manual/ blandaltman.php

19. Tronstad C, Pripp H. Statical methods for bioimpedance analysis. J Elect Bioimp 2014; 5: 14-27.

20. Quesada L, León C, Betancourt J, Nicolau E. Elementos teóricos y prácticos sobre la bioimpedancia eléctrica en salud. Rev Arch Med Camagüey 2016; 20 (5): 565-78.

21. Cáceres D, Messagi-Sartor M, Rodríguez D, Escalada F, Gea J, Orozco-Levi M, et al. Variabilidad de la composición corporal medida con bioimpedanciometría eléctrica según condiciones de realización: influencia del ayuno y del reposo. Nutr Hosp 2014; 30 (6): 1359-65.

22. Kotler DP, Burastero S, Wang J, Pierson Jr RN. Prediction of body cell mas, fat free mass and total body waterwith biolectrical impedance analysis: effects of race, sex and disease. AM J Clin Nutr 1996; 64: 489-97.

23. Björn J, Moritoyo T, Kaufer-Horwitz M, Peine S, Norman K, Maisch M, et al. Ethnic differences in fat and muscle mass and their implication for interpretation of bioelectrical impedance vector analysis. Appl Physiol Nutr Metab 2019; 44: 619-26.

24. CasasYG, Schiller BC, DeSouza CA, Seals DR. Total and regional body composition across age in healthy hispanic and White women of similar socioeconomic status. AM J Clin Nutr 2001; 73: 113-18.

25. Schifferli I, Lillo M. Behavior of a new equation using bioimpedanciometry to predict body fat in chilean adults. Obes Control Ther 2017; 4 (2): 1-7.

26. Bosy-Westphal A, Schautz B, Later W, Kehayias JJ, Gallager D, Müller MJ. What makes a BIA equation unique? Validity of eight-electrode multifrecuency BIA to estimate body composition in a healthy adult population. Eur J Clin Nutr 2013; 67: S14-S21; doi: 10.1038/ ejcn.2012.160.

27. Jensen B, Moritoyo T, Kaufer-Horwitz M, Peine S, Norman K, Maisch M, et al. Ethnic differences in fat and muscle mass and their implication for interpretation of bioelectrical impedance vector analysis. Appl Physiol Nutr Metab 2019; 44: 619-26. 